\title{
Captação de recursos para pesquisas e o terceiro setor: o que os docentes sabem?
}

\author{
Research fundraising and third sector organizations: what do the professors know?
}

\author{
Sabrina Brabo de Araújo Carvalho' (1) | sabrinabrabo@hotmail.com \\ Charles Alberto Villacorta de Barros ${ }^{1}$ (D) charles_villacorta@hotmail.com \\ Livia Gomes Limonge1 (D) llivia221@gmail.com \\ Andrey de Almeida Carneiro 2 (D) andreyalmeidac@gmail.com \\ Bertho Vinicius Rocha Nylander ${ }^{3}$ (D) berthonylander92@gmail.com \\ Paula Lavigne de Sousa Costa ${ }^{2}$ (D) paulalavigne9@hotmail.com
}

\begin{abstract}
RESUMO
Introdução: O crescimento socioeconômico de um país acontece por meio do desenvolvimento de ciência e tecnologia, e, por isso, a parceria com o terceiro setor é positiva ao auxiliar o financiamento de projetos científicos.

Objetivos: Assim, o presente estudo objetiva avaliar as formas de captação de recursos mais utilizadas pelos docentes de Medicina para a execução de seus estudos científicos e verificar se há o reconhecimento do terceiro setor como opção para tal obtenção.

Métodos: Trata-se de um estudo transversal analítico com abordagem quali-quantitativa, construído por meio do uso de um formulário individual aplicado aos docentes do curso médico de cinco instituições do ensino superior do Norte/Nordeste do Brasil. Os dados obtidos foram analisados por estatísticas descritiva e analítica.

Resultados: Participaram 138 professores, de ambos os sexos, com idade média \pm 43,2 anos. A pesquisa mais comumente desenvolvida pelos docentes foi a básica (56,1\%); 68,8\% utilizaram recursos próprios para o desenvolvimento de seus estudos; $18,8 \%$ obtiveram recursos de entidades de apoio à pesquisa; $75,4 \%$ conheciam alguma fundação de amparo à pesquisa, e as mais citadas foram a Fapespa (26,9\%) e o CNPq (26,9\%); $13,8 \%$ relataram conhecer sites e empresas multinacionais que fomentam projetos de pesquisas, mas somente $2,2 \%$ submeteram sua iniciativa às chamadas públicas em instituições internacionais para captação; quando perguntados sobre o terceiro setor, seus fundamentos e atores definidos pela legislação, o estudo apontou uma falta de conhecimento, com 100\% de inadequação nas respostas sobre tais entidades; quanto à parceria entre o terceiro setor e o Estado, 83,3\% sinalizaram desconhecimento; $100 \%$ dos entrevistados desconhecem os critérios para uma entidade integrar tal setor, ao mesmo tempo que $76,8 \%$ afirmaram que a falta de orientação dos conceitos do ramo não é entrave para a captação de recursos às pesquisas.
\end{abstract}

Conclusão: A maioria dos docentes utilizava recursos próprios para a realização de seus projetos, seguidos pelo uso de recursos públicos. Ademais, grande parte dos profissionais não reconheceu o terceiro setor como fonte patrocinadora, carecendo de informações que lhes possibilitassem desenvolver atividades de forma ampla e com as diversas oportunidades existentes, oferecidas por entidades desse setor.

Palavras-chave: Organizações Não Governamentais; Recursos Financeiros em Saúde; Pesquisa; Projetos de Pesquisa; Financiamento da Pesquisa.

\begin{abstract}
Introduction: The socio-economic growth of a country is reflected by the development of science and technology. Partnership with the third sector is therefore positive in terms of financial support for scientific projects.

Objective: Accordingly, the present study aims to evaluate the most commonly used methods of fundraising by medical professors to conduct their scientific studies and whether there is recognition of the third sector as an option for such fundraising.

Method: A cross-sectional analytical study with a qualitative and quantitative approach. The data were collected using an individual form, applied to medical course professors from five different universities in the north of Brazil, and analyzed using descriptive-analytical statistics.

Results: The study included 138 professors, of both sexes, with an average age of \pm 43.2 years. The type of research most commonly performed by professors was basic (56.1\%); $68.8 \%$ used their own resources to develop their studies; 18.8\% obtained funds from research support entities; $75.4 \%$ knew of a research support foundation, primarily FAPESPA (26.9\%) and CNPQ (26.9\%); $13.8 \%$ reported knowledge of websites and multinational companies that promote research projects, but only $2.2 \%$ submitted their initiative to public calls for funding from international institutions; when asked about the third sector, its fundamentals and actors defined by legislation, the study indicated a lack of knowledge, with $100 \%$ of inadequate answers about such entities; regarding partnership between the third sector and the State, $83.3 \%$ signaled ignorance; $100 \%$ of the interviewees were unaware of the criteria for an entity to be part of the sector, while $76.8 \%$ stated that lack of orientation about the concepts of the area is not an obstacle to raising funds for research.
\end{abstract}

Conclusion: Most professors use their own funds to carry out their projects, followed by the use of public funds. Furthermore, most professionals did not recognize the third sector as a source of sponsorship, lacking information that would allow them to develop broad activities and enjoy the various opportunities offered by entities in this sector.

Keywords: Non-Governmental Organizations; Financial Resources in Health; Research; Research Design; Research Funding.

${ }^{1}$ Universidade do Estado do Pará, Belém, Pará, Brasil.

${ }^{2}$ Faculdade Metropolitana da Amazônia, Belém, Pará, Brasil.

${ }^{3}$ Centro Universitário do Estado do Pará, Belém, Pará, Brasil.

Editora-chefe: Rosiane Viana Zuza Diniz ～～Editor associado: Kristopherson Lustosa Augusto

Recebido em 16/10/20; Aceito em 21/02/21. | Avaliado pelo processo de double blind review. 


\section{INTRODUÇÃO}

O crescimento socioeconômico de um país acontece por meio do desenvolvimento de ciência e tecnologia vinculadas à educação qualificada, de forma a fortalecer o polo industrial local. Nesse sentido, é imperiosa a maior promoção da produção científica, a fim de que sua base se torne social e economicamente sólida e robusta ${ }^{1,2}$.

Nos últimos anos, vem ocorrendo uma expansão numérica da produção brasileira decorrente do trabalho desenvolvido pelo Estado, destacadamente por meio da Coordenação de Aperfeiçoamento de Pessoal de Nível Superior (Capes) com apoio à pós-graduação ${ }^{1,2}$

Entretanto, a edição do Global Innovation Index 2019, ranking que apresenta as economias mais inovadoras do mundo, aponta o Brasil ainda na $66^{\mathrm{a}}$ posição, o que não é compatível com a nona economia mundial e sua própria grandeza territorial ${ }^{3,4}$.

Ressalta-se o aumento do número de pesquisadores brasileiros em busca de bolsas e de apoio financeiro para seus estudos, contudo tal fato ocorre em um contexto no qual a participação e o incentivo do Estado para financiá-los diminuíram, o que levanta a pergunta: "Quem proverá o recurso financeiro para o desenvolvimento científico em nosso país?"2,5,6.

Em países desenvolvidos, a solução encontrada para promover as áreas de tecnologia e de inovação foi a parceria com o terceiro setor como agente financiador de projetos nos âmbitos da saúde, da educação e da pesquisa, tornando-se uma prática comum e de longa data ${ }^{7}$.

Mas, para entender o papel do terceiro setor, é necessário contextualizá-lo. A partir de um conceito econômico, a sociedade é dividida em três setores: o primeiro setor corresponde à administração pública direta e indireta, com suas organizações pertencentes ao Estado, incluindo empresas e fundações públicas. O segundo setor é conhecido como o mercado, representando as atividades que perseguem o lucro, a produtividade e a criação de empregos ${ }^{8,9}$.

As entidades de interesse social sem fins lucrativos, popularmente conhecidas como organizações nãogovernamentais (ONGs), constituem o terceiro setor. Essas entidades captam recursos no mercado e são capazes de desenvolver atividades, tornandose parceiras do Estado. Uma vez apoiadas por capital estrangeiro ou nacional, nos setores social e educacional, representam uma importante alternativa para realização de ações transformadoras em diversas áreas, inclusive na pesquisa, na ciência e na tecnologia. A experiência com o terceiro setor é de grande valia no Reino Unido, Canadá, entre outros países, e ele tem ampliado suas atividades no mercado brasileiro ${ }^{10}$.

Dessa forma, este estudo tem como principais objetivos avaliar as formas de captação de recursos mais utilizadas pelos docentes do curso de Medicina para a execução de suas pesquisas científicas e verificar se há o reconhecimento do terceiro setor como opção para tal obtenção.

\section{MÉTODO}

Realizou-se um estudo transversal, analítico, de natureza experimental, com abordagem quali-quantitativa, aprovado em Comitê de Ética em Pesquisa (CEP) sob número 3.127.360, a partir da aplicação presencial de um formulário aos docentes incluídos na pesquisa, para esclarecimento e apresentação do Termo de Consentimento Livre e Esclarecido (TCLE), por meio do Google Forms.

Como critério de inclusão, os participantes deveriam ser docentes do quadro funcional de uma instituição de ensino superior (IES) pública e/ou privada, ativos, lotados no curso de Medicina, com tempo mínimo de atuação de três anos, ainda que em instituições diferentes. A aplicação dos formulários deuse no período de fevereiro a junho de 2019 em cinco IES: Centro Universitário Metropolitano da Amazônia (Unifamaz), Universidade do Estado do Pará (Uepa), Centro Universitário do Estado do Pará (Cesupa), Universidade Federal do Maranhão (Ufma) e Universidade Federal do Pará (Ufpa), a partir da anuência por parte da instituição sobre a realização da pesquisa em seu câmpus.

Foi realizada a construção de um formulário aplicado aos docentes constituído de três blocos. A primeira parte possuía seis perguntas de múltipla escolha e analisou o perfil sociodemográfico dos participantes com perguntas sobre idade, sexo, cadastramento na Plataforma Brasil, anos de docência, orientação em trabalho científico, instituição em que tem vínculo laboral e renda.

O segundo bloco do formulário, formado por 16 perguntas, discursivas e de múltipla escolha, questionou a produção científica do pesquisador, qual o tipo de estudo que realiza/realizou, se é ou foi orientador de alguma pesquisa, qual a média de custo das iniciativas desenvolvidas e se utilizou recurso próprio ou financiamento para que estas se desenvolvessem, além de seu conhecimento acerca de possíveis editais que apoiassem a publicação científica.

O terceiro e último bloco apresentava seis questões de múltipla escolha sobre o conceito de terceiro setor, a sua forma e a área de atuação, além das instituições que o integram, baseado no Marco Regulatório do Terceiro Setor, Lei no 13.019/2014.

Os dados coletados foram apurados em banco de dados elaborado no software Microsoft Office Excel 2013 para caracterização amostral. Para análise, aplicou-se estatística descritiva, com construção de tabelas e gráficos para apresentação dos resultados, e calcularam-se as medidas de posição e dispersão, como média aritmética e desvio padrão. 
O teste de Shapiro-Wilk foi o escolhido para realizar a estatística analítica, a fim de avaliar a normalidade das variáveis. As variáveis categóricas da amostra foram estudadas por meio dos seguintes testes: qui-quadrado e $\mathrm{G}$ aderência (para tabelas univariadas) e $\mathrm{G}$ e qui-quadrado independência (para tabelas bivariadas). Aplicou-se análise de correlação para avaliar a associação entre as variáveis sociodemográficas e a atuação docente em pesquisa com o conhecimento da ação do terceiro setor no apoio à ciência.

As estatísticas descritiva e analítica foram realizadas no software BioEstat 5.3. Para a tomada de decisão, adotou-se o nível de significância $a=0,05$ ou $5 \%$, sinalizando com asterisco os valores significantes.

\section{RESULTADOS}

Os participantes da pesquisa totalizaram 138 docentes dos cursos de Medicina, sendo 37,0\% deles do Unifamaz, 34,8\% da Uepa, $26,8 \%$ do Cesupa, $14,5 \%$ da Ufpa e $13,8 \%$ da Ufma. Eles apresentavam uma idade média de 43,2 anos e 55,1\% eram do sexo feminino; em relação à titulação, 30,4\% eram doutores; $37,7 \%$, mestres; e 31,9\%, especialistas, com predominância de renda mensal acima de dez salários mínimos (79,7\%) (Tabela 1).

Dos docentes incluídos na pesquisa, cerca de 78,3\% dos pesquisadores já foram orientadores de trabalhos científicos e em torno de 92,8\% deles possuem cadastro na Plataforma Brasil. Quanto aos projetos produzidos nos últimos cinco anos, a maioria dos docentes gerou em torno de um a três estudos (48,6\%).

Ademais, foi possível observar que a quantidade de artigos científicos publicados possuiu maior frequência nos últimos
Tabela1. Perfil sociodemográfico dos participantes da pesquisa

\begin{tabular}{|c|c|c|}
\hline Perfil sociodemográfico & Freq. & $\%(N=138)$ \\
\hline \multicolumn{3}{|l|}{ Sexo } \\
\hline Feminino & 76 & $55,1 \%$ \\
\hline Masculino & 62 & $44,9 \%$ \\
\hline \multicolumn{3}{|l|}{ Faixa etária } \\
\hline$<30$ & 11 & $8,0 \%$ \\
\hline De 30 a 39 & 40 & $29,0 \%$ \\
\hline De 40 a 49 & 48 & $34,8 \%$ \\
\hline De 50 a 59 & 21 & $15,2 \%$ \\
\hline De 60 a 69 & 12 & $8,7 \%$ \\
\hline Sem informação & 6 & $4,3 \%$ \\
\hline Mínimo/média \pm DP/máximo & \multicolumn{2}{|c|}{$25 / 43,2 \pm 10,3 / 67$} \\
\hline \multicolumn{3}{|l|}{ Titulação máxima } \\
\hline Doutor & 42 & $30,4 \%$ \\
\hline Mestre & 52 & $37,7 \%$ \\
\hline Especialista & 44 & $31,9 \%$ \\
\hline \multicolumn{3}{|l|}{ Orientador de trabalho científico } \\
\hline Sim & 108 & $78,3 \%$ \\
\hline Não & 30 & $21,7 \%$ \\
\hline \multicolumn{3}{|l|}{ Cadastro na Plataforma Brasil } \\
\hline Sim & 128 & $92,8 \%$ \\
\hline Não & 10 & $7,2 \%$ \\
\hline \multicolumn{3}{|c|}{ Renda mensal em salários mínimos (SM) } \\
\hline Menor que 8 & 5 & $3,6 \%$ \\
\hline De 8 a 10 & 23 & $16,7 \%$ \\
\hline Mais de 10 & 110 & $79,7 \%$ \\
\hline
\end{tabular}

Tabela 2. Dados acerca do desenvolvimento de pesquisas e financiamento de projetos dos participantes

\begin{tabular}{|c|c|c|}
\hline Desenvolvimento de projetos e financiamento & Freq. & $\%(N=138)$ \\
\hline Utilizou recursos próprios nos projetos de pesquisa desenvolvidos. & 95 & $68,8 \%$ \\
\hline Utilizou recursos de entidades de apoio à pesquisa. & 26 & $18,8 \%$ \\
\hline \multicolumn{3}{|l|}{ Quais fundações de amparo à pesquisa conhece? } \\
\hline Fapespa & 103 & $99,0 \%$ \\
\hline Capes & 35 & $33,7 \%$ \\
\hline Outras & 26 & $25,0 \%$ \\
\hline Participou de algum edital para receber auxílio na publicação científica. & 34 & $24,6 \%$ \\
\hline Participou de algum edital que auxilia na participação de eventos científicos. & 42 & $30,4 \%$ \\
\hline CNPq & 7 & $26,9 \%$ \\
\hline Outras & 11 & $42,3 \%$ \\
\hline Sem informação & 1 & $3,8 \%$ \\
\hline
\end{tabular}


dois anos (26,8\%) e no mesmo ano da pesquisa (24,6\%). Desses estudos, de dois a cinco $(28,6 \%)$ foram trabalhos oriundos de pesquisas individuais ou em equipe realizadas pelo pesquisador.

O tipo de estudo mais comumente desenvolvido pelos pesquisados foi a pesquisa básica $(56,1 \%)$. A maioria dos docentes $(66,7 \%)$ declarou sua posição como orientador durante o período da pesquisa, enquanto $13,8 \%$ dos abordados afirmaram que nunca exerceram esse papel. $\mathrm{O}$ custo médio dos projetos empreendidos foi da ordem de até cinco mil reais em sua maioria (72,7\%).

Observou-se que a minoria (18,8\%) do corpo docente teve seus projetos financiados nos últimos cinco anos, e, entre os que obtiveram tais recursos, 26,9\% captaram da Fundação Amazônia de Amparo a Estudos e Pesquisas (Fapespa), 26,9\% do Conselho Nacional de Desenvolvimento Científico e Tecnológico (CNPq) e 42,3\% de outras instituições. Nesse sentido, destaca-se que $68,8 \%$ dos pesquisadores utilizaram recursos próprios nos projetos desenvolvidos, de maneira isolada ou complementando o recurso financiado.

No que se refere à participação de editais pelo docente para recebimento de auxílio financeiro para publicação científica, 75,4\% não registraram participação. Sobre editais para eventos científicos, 69,6\% também não alegaram atuação. Apesar desses achados, destaca-se que $75,4 \%$ deles conheciam fundações de amparo à pesquisa, como a Fapespa $(99,0 \%)$ e o CNPq (35,6\%), já que os participantes eram capazes de informar quando mais de uma empresa era conhecida.

No cenário que abrange as iniciativas que fomentam projetos de pesquisa, apenas $13,8 \%$ dos pesquisadores possuíam conhecimento acerca da existência de empresas multinacionais e/ou sites com esse intuito. Nesse sentido, as empresas mais conhecidas pelos participantes foram as privadas $(57,9 \%)$ e as farmacêuticas (36,8\%), sendo também citadas a Fundação Bill e Melinda Gates (10,5\%) e outras (36,8\%), em um cenário no qual os pesquisadores poderiam citar vários empreendimentos.

Em relação às chamadas públicas de instituições multinacionais, $97,8 \%$ dos pesquisados não submeteram projetos de pesquisa. Dos 2,2\% que obtiveram essa oportunidade, os participantes destacaram as empresas UGR, National Institutes of Health (NIH-Brasil), NIH-CNPq, Fundação Hermann Hering e Wells Fargo.

Por fim, o corpo docente foi questionado sobre conceitos básicos do terceiro setor, quem o compõe e quais os critérios para uma entidade ser considerada sem fins lucrativos. Nesse item, os docentes não obtiveram êxito, pois $100 \%$ dos participantes desconheciam tais informações. Em relação à atuação desse setor, $68,1 \%$ dos pesquisadores reconheceram sua função, porém, ao serem indagados sobre a parceria das entidades com o Estado, 83,3\% desconheciam esse aspecto (Tabela 3).

Ademais, 76,8\% dos docentes responderam que a falta de orientação sobre os conceitos do terceiro setor não se constitui

Tabela 3. Dados acerca do financiamento e fomento à pesquisas por iniciativas diversas

\begin{tabular}{lcc}
\hline \multicolumn{1}{c}{ Fomento à pesquisa } & Freq. & $\%(\mathbf{N}=\mathbf{1 3 8})$ \\
\hline $\begin{array}{l}\text { Já teve a oportunidade de submeter algum projeto de pesquisa a chamadas públicas de } \\
\text { instituições multinacionais. }\end{array}$ & 3 & $2,2 \%$ \\
Conhece empresas multinacionais ou sites que fomentam projetos de pesquisa. & 19 & $13,8 \%$ \\
Quais dessas empresas/sites você conhece? & & $\mathrm{N}=19$ \\
Empresas privadas & 11 & $57,9 \%$ \\
Empresas farmacêuticas & 7 & $36,8 \%$ \\
Fundação Bill e Melinda Gates & 2 & $10,5 \%$ \\
\hline
\end{tabular}

Tabela 4. Avaliação do conhecimento do docente acerca do terceiro setor

\begin{tabular}{lcc}
\hline \multicolumn{1}{c}{ Avaliação do conhecimento } & \% Adequado & \% Inadequado \\
\hline Atuação da organização social na busca de recursos e investimentos contínuos para pesquisa. ${ }^{*}$ & $80,4 \%$ & $19,6 \%$ \\
A atuação do terceiro setor é:* & $68,1 \%$ & $31,9 \%$ \\
A falta de orientação sobre os conceitos do terceiro setor é um entrave para o pesquisador na & & $76,8 \%$ \\
captação de recursos.** & $23,2 \%$ & $83,3 \%$ \\
Sobre a relação entre o terceiro setor e o Estado.** & $16,7 \%$ & $100,0 \%$ \\
Quem faz parte do terceiro setor.** & $0,0 \%$ & $100,0 \%$ \\
Critérios para que as instituições do terceiro setor sejam consideradas sem fins lucrativos.** & $0,0 \%$ & \\
\hline
\end{tabular}

${ }^{*} p<0,05$ para acertos $e^{* *} p<0,05$ para erros. 
num entrave para o pesquisador na captação de recursos. Em contrapartida, $80,4 \%$ afirmaram que toda pesquisa precisa de recursos e de investimentos contínuos e que o pesquisador acredita que a atuação de uma organização social pode ajudar a promover esse desenvolvimento.

\section{DISCUSSÃO}

O perfil sociodemográfico apresentou docentes, em sua maioria, do sexo feminino $(55,1 \%)$ e na faixa etária entre 40 e 49 anos (34,8\%). Observou-se que os resultados foram ao encontro de outras pesquisas sobre o mesmo perfil ${ }^{11}$. Quanto à titularidade, a média de mestres e doutores foi menor quando comparada a outros estudos, sendo $37 \%$ da amostra constituída por mestres e 30,4\% por doutores. Diversos fatores podem justificar tal resultado, a exemplo da existência predominante de programas de pós-graduação na Região Sudeste do país em comparação ao Norte e Nordeste, e de algumas faculdades pesquisadas serem jovens em seu funcionamento ${ }^{12}$.

Nos últimos cinco anos, cerca de $48,6 \%$ dos docentes produziram de um a três estudos, $78,3 \%$ foram orientadores de trabalhos científicos e 92,8\% eram cadastrados na Plataforma Brasil. Esse cenário ratifica que, apesar da falta de incentivo externo à produção científica, expresso por meio de editais de financiamento conhecidos pelo pesquisador, o profissional ainda busca produzir com o propósito de aumentar numericamente seus produtos ${ }^{13}$.

É nítido o crescimento da produção científica brasileira com o passar dos anos, o que pode ser confirmado pelas publicações qualificadas e indexadas no Institute for Scientific Information (ISI, Filadélfia, Estados Unidos). Durante a década de 1960, o Brasil publicava anualmente 52 artigos científicos, porém, durante a década de 2000, registraram-se mais de dez mil estudos anuais. Assim, o país passou a integrar a lista das 30 nações que mais publicaram no mundo. Contudo, questiona-se a qualidade da produção, pois os dados apresentados demonstraram que a pesquisa, principalmente médica e biomédica, no Brasil obteve um progresso exponencial no componente quantitativo, entretanto, no que cerne ao progresso qualitativo, existem apenas destaques pontuais ${ }^{14}$.

O tipo de pesquisa mais comumente desenvolvida foi a básica com 56,1\%, seguida da aplicada com 25,8\%; o custo médio de projetos apresentados ficou na ordem de cinco mil reais $(72,7 \%)$, e $68,8 \%$ dos pesquisadores utilizaram recursos próprios em seus estudos. Tais realidades podem ser justificadas pelos recursos escassos disponibilizados à produção científica, pois, nos últimos anos, houve, por parte do Estado, uma desresponsabilização em determinadas áreas, como a pesquisa, ao mesmo tempo que houve o aumento do incentivo e da participação da sociedade no desenvolvimento de políticas públicas para saúde e educação ${ }^{15,16}$.

Além disso, a burocracia que envolve o processo de captação de recursos é um obstáculo para o pesquisador que necessita aprender a lidar com todos os trâmites ${ }^{17}$. Nesse sentido, também é necessário analisar a hipótese de que o tempo de existência das instituições de ensino pode influenciar no desenvolvimento de iniciativas na área, havendo muitas faculdades particulares ainda jovens que vivem o desafio de ampliar sua captação de recursos para além da cobrança de suas mensalidades ${ }^{2}$.

Como o tamanho e a qualidade das pesquisas sofrem influência direta da metodologia, no dia a dia do pesquisador, o tamanho das dependências do laboratório e suas limitações estarão ligados proporcionalmente aos recursos, e estes, normalmente, são dispendiosos. Portanto, a parceria com o terceiro setor como forma de captar recursos é de grande valia, visto que as entidades ligadas a ele têm liberdade administrativa, o que diminui os aspectos burocráticos, mesmo que elas sofram fiscalização estatal ${ }^{18}$. Tal parceria é uma realidade recorrente em vários países, e, no Brasil, sua atividade tem crescido e já apresenta impacto no Produto Interno Bruto (PIB) nacional ${ }^{4}$.

Quanto à maioria dos trabalhos desenvolvidos, eles se concentraram na área da pesquisa básica. Esse tipo de estudo tende a ser desenvolvido por docentes não médicos, quando dedicado apenas à docência, e pelo médico atuante no ensino superior, quando destinado à área clínica ${ }^{19,20}$. Destaca-se que as ações das organizações sociais na sociedade podem contribuir para a mudança desse perfil da pesquisa brasileira, focada na área básica. Com o investimento de capital, há possibilidade de maior incentivo à pesquisa aplicada, o que promove o desenvolvimento social e econômico do país pela inovação tecnológica ${ }^{21,22}$.

Em relação à taxa de financiamento dos projetos desenvolvidos pelos docentes nos últimos cinco anos, 18,8\% conseguiram apoio financeiro, e a maioria $(68,8 \%)$ utilizou recursos próprios para o financiamento de seu projeto de pesquisa em algum momento de seu desenvolvimento. Os dados encontrados foram menores do que os constatados em outra pesquisa, no qual foram incluídos docentes de uma IES estadual do Nordeste, em que $30,8 \%$ dos projetos relatados pelos 246 profissionais obtiveram financiamento, dos quais $28,3 \%$ contaram com duas ou mais fontes ${ }^{23}$.

Sobre as fundações de amparo à pesquisa que mais financiaram os docentes do estudo, destacam-se a Fapespa $(26,9 \%)$ e o CNPq (26,9\%). Uma das hipóteses para ratificar esse fato é a existência de editais institucionalizados, o que os torna mais acessíveis ao docente por seu alcance regional. A participação em outros editais depende de ampla 
divulgação, além do fato de que a adesão aos processos exige acompanhamento frequente de seu lançamento, o que demanda tempo e preparação para submissão do projeto ${ }^{24}$.

Apesar disso, é importante notar que o financiamento à produção científica mais comumente disseminado pelas faculdades é aquele advindo de origem governamental, destinado ao desenvolvimento de ciência e tecnologia (CNPq) e às fundações de amparo à pesquisa (FAPs), entretanto mesmo essas entidades enfrentam o aumento da demanda por docentes interessados em orientar bolsistas nos programas, além da distribuição heterogênea das bolsas pelo país e dentro das universidades ${ }^{25}$.

A maioria das instituições que financiaram as pesquisas $(18,8 \%)$ era de natureza pública, o que mostra o Estado como principal meio de financiamento das pesquisas brasileiras. Esse fato pressupõe que o Brasil seja incipiente ainda na captação de recursos por meio de entidades que oferecem tal suporte, enquanto, em outros países, a prática quanto à participação do terceiro setor na promoção da pesquisa já é uma realidade ${ }^{26,27}$.

Ressalta-se também o valor investido - em torno de cinco mil reais - nas pesquisas, o que ratifica a importância de as entidades de ensino aumentarem seus investimentos no campo metodológico que é o propulsor de todo trabalho científico, a fim de transformarem o conhecimento produzido nas IES em produtos práticos na melhora da qualidade de vida da sociedade com geração de renda e riqueza ${ }^{27,28}$.

Dos pesquisados, 83,3\% desconheciam a real parceria entre o terceiro setor e o Estado, e a falta desse conceito básico não permite total discernimento das atividades desenvolvidas por tais entidades. Quando questionados sobre os atores que compõem esse segmento e sobre os critérios necessários para que as entidades possam ser consideradas desse setor econômico, o índice de erro apresentado foi de 100\%. Essa falta de conhecimento torna-se um entrave para o pesquisador na captação de recursos, sobretudo os atuantes na área de ciências biológicas, de forma que tais conceitos não são compartilhados no dia a dia e ficam em outras esferas do conhecimento ${ }^{29}$.

Apesar de $76,8 \%$ dos docentes afirmarem que a falta de orientação sobre os conceitos do terceiro setor não é um entrave para o pesquisador na captação de recursos, tal realidade é vista como um problema, pois, sem esse conhecimento, a captação de recursos fica restrita diante de um Estado que apresenta dificuldades em oferecer os devidos financiamentos à expansão das universidades e aos projetos científicos, o que aumenta a discussão da sustentabilidade econômica das IES brasileiras, tanto no ensino público como no privado 4 .

Grande parte dos docentes $(80,4 \%)$ afirmou que toda pesquisa precisa de recursos e de investimentos contínuos, e que o pesquisador acredita que a atuação de uma organização social pode ajudar a promover esse desenvolvimento. Nesse sentido, tal crença se consolidará como positiva quando estimulada perante os profissionais, com o propósito de que essa nova opção de arrecadação de recursos auxilie no quadro nacional de diminuição de gastos do Ministério de Ciência, Tecnologia, Inovação e Comunicações, assim como no maior contingenciamento de orçamentos direcionados à pós-graduação ${ }^{30}$.

O conhecimento sobre as formas de atuação e as entidades que compõem o terceiro setor deve ser estimulado, pois é fato que a participação estatal no apoio ao desenvolvimento das pesquisas sofreu uma diminuição, ao passo que políticas públicas são discutidas continuamente a fim de fomentar a atuação de empresas privadas e da própria sociedade para esse suporte.

\section{CONCLUSÃO}

A principal forma de recurso utilizada para realização de pesquisa pelos professores entrevistados foi o recurso próprio, seguido pelo uso de recursos públicos. Ademais, grande parte dos profissionais não reconheceu o terceiro setor como fonte patrocinadora, carecendo de informações que lhes possibilitassem desenvolver atividades de forma ampla e com as diversas oportunidades existentes oferecidas por entidades que compõem esse setor.

\section{AGRADECIMENTOS}

Agradecemos ao corpo docente do Programa de Cirurgia e Pesquisa Experimental (Cipe) da Universidade do Estado do Pará (Uepa) toda a contribuição científica e o apoio durante a realização da pesquisa; ao mestrando Dr. Cesar Collyer Carvalho pela aplicação do questionário nas instituições de ensino selecionadas e pela participação no projeto de pesquisa desde o seu início; à professora mestra Mariseth Carvalho Andrade que acompanhou e orientou todo o trabalho estatístico dessa pesquisa; e à professora mestra Bruna Pereira Carvalho Sirqueira que possibilitou a aplicação do formulário na Universidade Federal do Maranhão (Ufma).

\section{CONTRIBUIÇÃO DOS AUTORES}

Sabrina Brabo de Araújo Carvalho, Livia Gomes Limonge, Andrey de Almeida Carneiro, Bertho Vinicius Rocha Nylander e Paula Lavigne de Sousa Costa participaram da concepção e do design do estudo, da análise dos resultados e da escrita do artigo, com base na literatura atual e seguindo as coordenadas do orientador. Charles Alberto Villacorta de Barros orientou o estudo e revisou o artigo. Todos os autores aprovaram a versão final a ser publicada e são responsáveis por todos os aspectos do trabalho, incluindo a garantia de sua precisão e integridade. 


\section{CONFLITO DE INTERESSES}

Os autores declaram não haver conflito de interesses neste estudo.

\section{FINANCIAMENTO}

Não houve fomento externo para a pesquisa, e todos os gastos foram de responsabilidade dos pesquisadores.

\section{REFERÊNCIAS}

1. Santos SMC, Lima LS, Martelli DRB, Martelli-Júnior HM. Perfil dos pesquisadores da saúde coletiva no Conselho Nacional de Desenvolvimento Científico e Tecnológico. Physis. 2009;19(3):761-75.

2. Riscarolli V. Estratégias de captação de recursos aplicáveis à realidade das faculdades de administração de instituições de ensino superior brasileiras [tese]. São Paulo: Universidade de São Paulo; 2007.

3. Cornell University, Insead, Wipo. The Global Innovation Index 2019. Creating Heallthy Lives - The future of medical Innovation. 12th ed. Ithaca, Fontainebleau, Geneva: World Intellectual Property Organization and Confederation of Indian Industry; 2019.

4. Castro JMG. Apontamentos sobre a adoção das boas práticas de governança nas organizações do terceiro setor. Importância da adoção de um programa de compliance efetivo, à luz da Lei n $12.846 / 2015$. Quaestio luris. 2016;9(2):1012-30.

5. Mendonça FSF. A expansão do ensino superior e a contribuição das fundações privadas: um olhar do Ministério Público Estadual. Maceió: Cesmac, 2018.

6. Tachizawa T. Organizações não governamentais e terceiro setor: criação de ONGs e estratégias de atuação. São Paulo: Atlas, 2014.

7. Borges MN. As fundações estaduais de amparo à pesquisa e o desenvolvimento da ciência, tecnologia e inovação no Brasil. Revista USP. 2011;89:174-89.

8. Martins N, Thomazi A. Gestão social e desenvolvimento local: princípios, conceitos e possíveis relações com o terceiro setor. Revista Tecer. 2014;7(12):15-22.

9. Peroni V, Oliveira R, Fernandes M. Estado e terceiro setor: as novas regulações entre o público e o privado na gestão da educação básica brasileira. Educ Soc. 2019;30(108):761-78.

10. Hardwick R, Anderson R, Cooper C. How do third sector organizations use research and other knowledge? A systematic scoping review. Implement Sci. 2015;10:84.

11. Vieira MHP, Fontes ARM, Gemma SFB, Montedo UB. Produtivismo na pós-graduação na perspectiva da ergonomia da atividade. Educ Pesqui. 2020;46:e220223.

12. Novaes HMD, Werneck GL, Cesse EAP, Goldbaum M, Minayo MCS. Pósgraduação senso estrito em Saúde Coletiva e o Sistema Único de Saúde. Cien Saude Colet. 2018;23(6):2017-25.

13. Barbosa DMM, Gutfilen B, Gasparetto EL, Koch HA. Análise do perfil dos egressos do Programa de Pós-Graduação em Medicina (Radiologia) da Faculdade de Medicina da Universidade Federal do Rio de Janeiro. Radiol Bras. 2009;42(2):121-4
14. Guimarães JA. A pesquisa médica e biomédica no Brasil: comparações com o desempenho científico brasileiro e mundial. Cien Saude Colet. 2004; 9(2):303-27.

15. Gomes LA, Brasileiro TSA. Políticas educacionais: as marcas da descentralização. Cad Pesqui (São Luís). 2018;25(2):33-52.

16. Hanauer MJ, Abegg I. Políticas públicas e programas de incentivo à integração de tecnologias no ensino. Jornal de Políticas Educacionais. 2017;11(16):1-18.

17. Chiariello $\mathrm{CL}$, Bernardes ALT. Empreendedorismo nas estratégias de captação de recursos financeiros das APAEs de Campo Grande, Dourados e Três Lagoas-MS. Revista Livre de Sustentabilidade e Empreendedorismo. 2017;2(4):165-82.

18. Vieira NS, Parente C, Barbosa ACQ. "Terceiro setor", "economia social" e "economia solidária": laboratório por excelência de inovação social. Sociologia: Revista da Faculdade de Letras da Universidade do Porto. 2017; (Número temático - Processos sociais e questões sociológicas):100-121.

19. Almeida MJ. Ensino médico e o perfil do profissional de saúde para o século XXI. Interface (Botucatu). 1999;3(4):123-32.

20. Garcia MAA, Silva ALB. Um perfil do docente de medicina e sua participação na reestruturação curricular. Rev Bras Educ Med. 2011;35(1):58-68.

21. Fleury MTL, Werlang SRC. Pesquisa aplicada: conceitos e abordagens. GV Pesquisa. 2016:10-5.

22. Morais HMM, Albuquerque MSV, Oliveira RS, Cazuzu AKI, Silva NAF. Organizações sociais da saúde: uma expressão fenomênica da privatização da saúde no Brasil. Cad Saude Publica. 2018;34(1):e00194916.

23. Santos FGPL, Kovacs $M H$, Melo RAM, Beltrão $R C l$, Santiago RRS Alencar AS, et al. Produção científica dos docentes do curso médico da Universidade de Pernambuco - um estudo exploratório. Rev Bras Educ Med. 2005;29(2):81-90.

24. Silva Neto NC, Lopes SF, Torres JC, Brandão CF. A inserção da CAPES na formação de professores na educação básica no Brasil. EccoS Revista Científica. 2016;(40):145-61.

25. Freitas AG, Zarzar PMPA, Paiva SM, Pordeus IA, Ferreira FM. Perfil dos orientadores de bolsistas de iniciação científica da Faculdade de Odontologia da Universidade Federal de Minas Gerais. Arq Odontol. 2020;56:e03.

26. Mañas AV, Medeiros EE. Terceiro setor: um estudo sobre a sua importância no processo de desenvolvimento sócio-econômico. Perspectivas em Gestão \& Conhecimento. 2012;2(2):15-29.

27. Martins LEG. A importância de fundos patrimoniais em universidades [tese]. Rio de Janeiro: Universidade Federal do Rio de Janeiro; 2020.

28. Aveni A, Carvalho SMS, Doroteu LR, Mello AS. O terceiro setor no Brasil e sua relação com a economia nacional. Negócios em Projeção. 2018;9(1):103-14

29. Falcão MA, Araujo RS. A importância estratégica do terceiro setor no Brasil como meio de desenvolvimento social: uma argumentação teórica a partir do prisma da economia social de Gide. Revista Jurídica Cesumar. 2017;17(1):153-79.

30. Costa ALL. A importância do conhecimento científico para as instituições de ensino superior. Rev Ciênc Plur. 2017;3(1):1-2. 